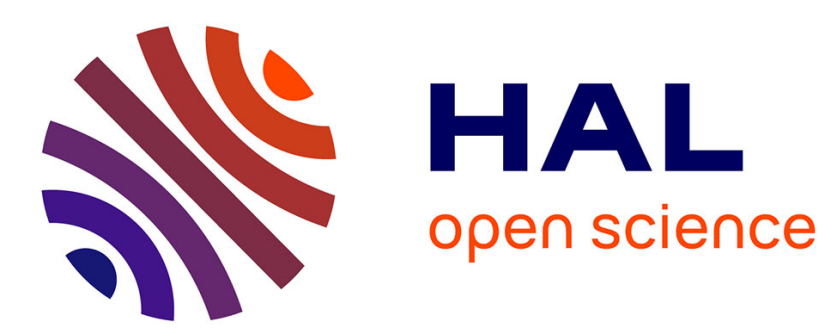

\title{
Study of the Turbulent Velocity Field in the Near Wake of a Bluff Body
}

Juan José Cruz Villanueva, Luís Fernando Figueira da Silva

\section{To cite this version:}

Juan José Cruz Villanueva, Luís Fernando Figueira da Silva. Study of the Turbulent Velocity Field in the Near Wake of a Bluff Body. Flow, Turbulence and Combustion, 2016, 97 (3), pp.715-728. 10.1007/s10494-016-9709-6 . hal-03313660

\section{HAL Id: hal-03313660 \\ https://hal.science/hal-03313660}

Submitted on 21 Aug 2021

HAL is a multi-disciplinary open access archive for the deposit and dissemination of scientific research documents, whether they are published or not. The documents may come from teaching and research institutions in France or abroad, or from public or private research centers.
L'archive ouverte pluridisciplinaire HAL, est destinée au dépôt et à la diffusion de documents scientifiques de niveau recherche, publiés ou non, émanant des établissements d'enseignement et de recherche français ou étrangers, des laboratoires publics ou privés. 


\title{
STUDY OF THE TURBULENT VELOCITY FIELD IN THE NEAR WAKE OF A BLUFF BODY
}

\author{
Juan José Cruz Villanueva \\ Luís Fernando Figueira da Silva \\ Pontifícia Universidade Católica do Rio de Janeiro, Department of Mechanical \\ Engineering, Rio de Janeiro, Brazil
}

Tel: +55 21 3527-1176; Fax +55 21 3527-1165

juancruz@aluno.puc-rio.br-luisfer@ puc-rio.br

\begin{abstract}
An experimental characterization of the turbulent flow structure formed downstream of a vertically mounted circular bluff body is performed. Three components of an instantaneous velocity field are measured using the stereo particle image velocimetry technique at the symmetry plane. The average velocity and the turbulent properties are analyzed. The results indicate a recirculation zone consisting of a toroidal vortex with similar dimensions for all Reynolds numbers. The largest turbulent fluctuations are found at the stagnation point region. The observed anisotropy of the normal Reynolds stress components is associated with the stagnation point flow, whereas the crosscorrelation component extreme occurs in high strain rate regions. An analysis of the Reynolds tensor anisotropy using the Lumley triangle is performed, revealing that the largest departures from isotropy occur at high shear regions and also within the vortex.
\end{abstract}

Keywords: PIV; wake flow; anisotropy; Reynolds stresses; Lumley triangle.

\section{Introduction}

Turbulent flow downstream bluff bodies, such as cylinders or circular discs, are widely employed for combustion stabilization [1] due to the formation of a recirculation zone at the vicinity of the obstacle face. In this recirculation, which is associated with the pressure drop at the near wake region, close to the face of the bluff body, fluctuating velocities and high strain rate fields are expected to occur. The interaction between these flow field characteristics and combustion controls the flame stabilization process. Thus, the description of the properties at the turbulent near wake region, in particular those controlled by the formation of large scale structures, is essential to an adequate understanding of the physical mechanisms that govern the combustion stabilization downstream of these obstacles. Indeed, in fuel/air shear layer large strain rate values may promote a partial premixing of the reactants, thus facilitating combustion. However, these large strain rates may also complicate the stabilization of non-premixed flames and cause local extinction of the combustion process. Therefore, a detailed flow field characterization of this near wake region is crucial to understanding the interplay between combustion, turbulence and mixing. 
This study aims to describe the turbulent isothermal flow properties and structure of the recirculation region downstream of a bluff body with a simple cylindrical geometry. To the best of the author's knowledge, a detailed characterization of turbulence anisotropy in such a self-confined near wake is absent from the literature. This is significant to combustion applications because this configuration has been used, for instance to stabilize non-premixed methane and ethanol-air flames at the bluff body recirculation zone [2,3]. Also, it is expected that the present results will eventually lead to an important of transported Probability Density Function Large Eddy Simulation models developed by the authors $[4,5,6]$.

Laser Doppler anemometry (LDA) has been used by Durao and Whitelaw [7] to measure the velocity field, turbulent properties and probability density distribution in the region of recirculating annular jets for different Reynolds numbers, $\operatorname{Re}=5,400$ to 36,000 . The turbulent fields studied were substantially anisotropic. The evolution of the shear layer issuing from the edge of a circular bluff body has been studied by Huang and Lin [8] for Reynolds numbers up to 2000. Concerning the flow structure, five characteristic modes have been found: laminar, subcritical, transitional, supercritical and turbulent. In these cases, flow patterns, such as vortex radius length and turbulent properties, have been shown to be closely related to the characteristic modes, even though slight variations in the position of the center and radius of the vortex have been evidenced for the supercritical and turbulent modes. Braza et al. [9] have characterized the near field wake of a circular cylinder in which the main axes are perpendicular to the flow direction, determining flow structures and Reynolds stresses. More recently [10], high-repetition-rate PIV measurements have been performed to study an annular recirculation region. The temporal analysis of the wake frequency spectrum indicated that periodic vortex shedding is absent.

The detailed characterization of the turbulent flow properties of planar wakes is available $[11,12]$. Additionally, starting from the pioneering work of Uberoi and Freymuth [13], recent studies $[10,14,15]$ address the axisymmetric wake behavior at large distances downstream of the bluff body. The turbulent characterization of five different bluff body flows realized by Antonia's group [16], shows that the inertial range scales are affect by the large scale anisotropy, which depend on the inflow conditions and on the bluff body shape. However, the small scales are not strongly affected by the wake generator shape, and the isotropy assumption results in a reasonable approximation in this range [17]. For the combustion process stabilization of interest to the authors [2,3], the knowledge of the near wake properties is essential. Nevertheless, to the best of the author's knowledge, the detailed characterization of turbulent anisotropy at the bluff body near the wake region has not been performed. The turbulent flow properties are assessed here using the trajectories in the Lumley triangle [18]. A similar assessment has been performed in stirred tanks [19,20], which involve a periodic, three-dimensional flow field.

This paper first presents a brief overview of the classical experimental methodology used and the associated uncertainty analysis. The studied flow fields are then compared by analyzing the averaged flow properties. The turbulent properties are discussed in detail with emphasis placed on the recirculation region anisotropy. 


\section{Methodology}

This section briefly describes the experimental apparatus, i.e., a classical bluff body burner, introduces the used stereo particle imaging velocimetry (SPIV) technique, and presents an experimental uncertainly analysis of the measurements.

\subsection{Experimental considerations}

The experiments are performed in the arrangement and are schematically illustrated in Fig. 1, which also shows the apparatus dimensions along with the measurement coordinates. A 60-mm diameter $\left(D_{b}\right)$ circular bluff body is located concentrically in a 200-mm diameter duct, which constitutes an annular wind tunnel. The verticality and alignment of the bluff body are regulated with precision screws located at the bluff body base. The ample optical access of this configuration allows for an easy application of laser diagnostics. A centrifugal fan, Deltra VC-400, with a maximum flow rate of $2.8 \mathrm{~m}^{3} / \mathrm{min}$ is used to supply air to the experiment. The minimum and maximum air flow velocities $\left(u_{\infty}\right)$ are approximately $4 \mathrm{~m} / \mathrm{s}$ and $11 \mathrm{~m} / \mathrm{s}$, respectively. The air supply system includes a tranquilizing chamber and circular perforated grids, upstream to the bluff body, which consists of 12 -mm holes separated at approximately $10 \mathrm{~mm}$ from each other, to provide uniform flow velocity and to generate turbulence.

\section{Figure 1}

The SPIV technique is used to study the near wake flow field corresponding to the different air flow velocities [21]. The dual laser pulse Nd:YAG CFR 200, $200 \mathrm{~mJ}$ of energy at $15 \mathrm{~Hz}$, provides a laser beam at the second harmonic ( $\lambda=532 \mathrm{~nm}$ ), which is transformed into a $0.5-\mathrm{mm}$ average thickness plane using a set of lenses. The time between laser pulses is adjusted to restrict the displacement of the particles between laser pulses to $5 \mathrm{px}$. The walls of the annular duct and bluff body have been painted black to reduce laser scattering. The images are focused by using Nikon lenses (Nikkop, $\mathrm{f} / 1.8 \mathrm{D}$ and $50 \mathrm{~mm}$ ), and the recording is made with two image intense CCD cameras (LaVision) with $1376 \mathrm{x} 1040 \mathrm{px}^{2}$ and $10 \mathrm{~Hz}$ for the maximum frame rate. DAVIS 7.2 software is used to control the trigger signal of lasers and cameras. Titanium oxide $\left(\mathrm{TiO}_{2}\right)$ tracer particles are homogeneously seeded in the air flow using a fluidized bed combined with a cyclone, thus providing particles with an average diameter of $1.0 \mu \mathrm{m}$. These diameters have been measured using a flight time spectrometer (TSI APS 3320). This particle diameter ensures good light scatter intensity and allows us to follow the flow fluctuations of up to $70 \mathrm{kHz}$. The cameras are arranged at an angle of $35^{\circ}$ and oriented towards the same side of the laser plane. The measurement region, $140 \times 105 \mathrm{~mm}^{2}$, is located downstream of the bluff body surface and centered on its symmetry line, thus leading to a $0.1 \mathrm{~mm} / \mathrm{px}$ image resolution. The particle displacement fields are obtained by applying multi-pass FFT decreasing window size of $32 \times 32$ to $16 \times 16 \mathrm{px}^{2}$ in three passes, with $50 \%$ overlap, resulting in an 
uniform grid size $\Delta x_{i}=1.15 \mathrm{~mm}$ spacing between velocity vectors. The Whittaker reconstruction [22] method is employed to achieve sub-pixel displacement resolution. Finally, average velocity fields, $u_{i}$, and Reynolds stresses, $\overline{u_{i}^{\prime} u_{j}^{\prime}}$, result from processing 4000 pairs of instantaneous images.

\subsection{Uncertainty analysis}

Even after decades of study [23], the quantification of experimental uncertainties associated with PIV measurements seems to be an open problem. Indeed, recent work [24] indicates that several uncertainty quantification techniques commonly used have not yet achieved a level of maturity that allows them to be considered as standards.

Indeed, these authors compare four different a posteriori uncertainty determination methods with respect to their ability to quantify known measurement errors. PIV uncertainty $\left(\varepsilon_{U}\right)$ is usually split into four major sources: (1) equipment $\left(\varepsilon_{E}\right),(2)$ particle dynamics $\left(\varepsilon_{P}\right),(3)$ sampling $\left(\varepsilon_{S}\right)$, and (4) image analysis $\left(\varepsilon_{A}\right)$ and total uncertainty is $\varepsilon_{U}=\sqrt{\varepsilon_{E}^{2}+\varepsilon_{P}^{2}+\varepsilon_{S}^{2}+\varepsilon_{A}^{2}}$. Quantifying the first of these sources is relatively straightforward because the equipment specifications are usually known beforehand and do not change when the experimental boundary conditions are modified. Typical values for this uncertainty lie below $1 \%$. The second uncertainty source is closely tied to experimental details, such as the type of flow being studied. In turbulent flows, sampling errors affect the accuracy with which statistical moments are to be reported. Typically, uncertainties dramatically increase for moments of order higher than two [25], and their values are closely dependent on the (unknown) statistical distributions of the velocity components. Finally, image analysis encompasses processing algorithm choices and their corresponding controlling parameters [26]. Quantifying this error source is a laborious task and usually involves generating synthetic particle images in several representative conditions. These representative conditions involve addressing errors associated with peak locking, insufficient seeding, displacement gradients within the interrogation window, window shifting, and out-of-plane particle movement, for instance.

The uncertainty analysis in the vortex region is present next, where $\varepsilon_{i}$ is given, as a percentage of local velocity. In the present experimental setup, equipment uncertainties $\varepsilon_{E}$ result from dimension calibration errors at the physical and image planes, from the distance between these planes, and from image distortion, and also from trigger synchronization and pulse timing inaccuracies. The total uncertainty associated with these sources is estimated to be of the order of $\varepsilon_{P}=1 \%$, being dominated by the calibration image plane length measurement and by image distortions.

The air is seeded with $\mathrm{TiO} 2$ particles generated by an ensemble fluidized bed and cyclone, with a measured diameter distribution between 0.6 and $1.5 \mu \mathrm{m}$. Using the methodology proposed by Melling [27], this ensures that velocity fluctuations up $5.4 \mathrm{kHz}$ could be followed by the particles, that $50 \%(<0.9 \mu \mathrm{m})$ of them would follow fluctuations up to $2.2 \mathrm{kHz}$ and that the Stokes number is 0.027. The use of this small diameter particle leads to a slip velocity between particles and the flow field, which is expected to be smaller than $\varepsilon_{P}=10^{-4} \%$. The out-of-plane particle displacement 
between laser pulses ( $d t=39 \mu \mathrm{s})$ is evaluated by multiplying this $\mathrm{dt}$ by the radial mean velocity. This displacement, expressed as a percentage of the laser plane thickness $(\sim 500 \mu \mathrm{m})$ is always smaller than $9 \%$.

The computation of velocity field statistical moments uses 4,000 vector fields. A convergence analysis of the velocity and Reynolds stress tensor components shows that increasing the number of used fields by $10 \%$ leads to a modification of the normal components, which is smaller than $\varepsilon_{S}=$ 0,6 and $1 \%$, respectively, in the sense of an Euclidean norm.

An analysis of the uncertainties associated with image processing for the present experiments using the latest statistical tools is outside the scope of the present work. The uncertainty contributions associated with image analysis have thus been characterized using the results obtained by Raffel $e t$ al. [21], which are considered to be representative of the procedures followed here. Imaging processing uses three passes with decreasing window size. The particle size at the image plane, which is influenced by refraction and magnification, leads to a $0.9 \%$ maximum uncertainty in particle displacement determination. The CCD camera noise level is smaller than 5\%, which implies a negligibly small influence on the uncertainty of the larger particle displacements. In large strain rate regions, the particle displacement gradient within an interrogation window may reach 0.04 and is expressed in pixel/pixel. Considering that the number of particles within a window is not smaller than 15 , this leads to a maximum uncertainty of $8 \%$, which occurs at the vicinity of the maximum shear stress region. Note that the typical value of this uncertainty within the flow field is smaller than $3 \%$. Considering that the particle images typically occupy two px, the subpixel corrections introduce a $0.5 \%$ uncertainty on particle displacement. Therefore image analysis uncertainty is $\varepsilon_{A}$ $=3.2 \%$ of local velocity.

Combining all these local uncertainty sources leads to a typical uncertainty of $\varepsilon_{U}=3.4 \%$ with respect to the local velocity and $\varepsilon_{U}=0.6 \%$ of full velocity scale. Note that in high shear regions, relative uncertainty values are locally higher, typically of the order of $10 \%$ of local mean velocity.

\section{Results and Discussion}

Three different air flow Reynolds numbers (based on the bluff body diameter $D_{b}$ and the freestream annular air velocity, $\boldsymbol{u}_{\infty}$ ) are initially considered: $15,000,30,000$ and 45,400 . Then, the analysis focuses on the latter case, which exhibits the higher turbulence Reynolds number, 1300, based on the measured turbulence intensity and the radius of the toroidal vortex. In this work, the turbulent integral length scale $L_{t}$ has been determined by using the spatial correlation coefficient [11]. The corresponding values calculated at the freely standing stagnation point are 12.3 and $8.8 \mathrm{~mm}$ along the longitudinal and radial directions, respectively. Therefore, the integral length scale is assumed to be proportional to a sixth of the bluff body diameter, $L_{t} \approx D_{b} / 6$. The turbulent intensity is taken to be $20 \%$ of free stream velocity, $u^{\prime} / u_{\infty} \approx 20 \%$, which will be shown below to be a typical measured value. Following a classical order of magnitude analysis [11], this leads to a turbulent 
Reynolds number of 1300 , and to a Kolmogorov length scale of the order of $46 \mu \mathrm{m}$. The Kolmogorov frequency is estimated to be $7.5 \mathrm{kHz}$.

Throughout this paper, all dimensions and velocities are non-dimensionalized by $D_{b}$ and $u_{\infty}$, spatial derivatives by $u_{\infty} / D_{b}$ and turbulent kinetic energy and anisotropy $a_{i j}$ by $u_{\infty}^{2}$, respectively. The three measured velocity components allow the determination, under the hypothesis at zero circumferential mean velocity derivate, that the continuity equation residual is smaller than $5 \%$. Furthermore, the obtained results show that symmetry with respect to the centerline $(r=0)$ is also verified. Indeed, the deviations observed are smaller than $2 \%$. Therefore, the flow field properties are presented in $(x, r)$ coordinates, with corresponding velocity components $u, v$.

In this section, first, the structure of the average velocity field is briefly presented, followed by an analysis of the average, strain rate and vorticity components. Turbulence is characterized by the anisotropy tensor components. Finally, the invariants in the Lumley triangle representation are examined.

\subsection{Average velocity field}

Figure 2 shows the average field of the velocity component in the longitudinal flow direction, $u$, and the corresponding flow structure. In Fig. 2, streamlines are superimposed on the average velocity field (left half), and the velocity vectors are given at the other half. Note the air flow convergence toward a stagnation point at the centerline $(r=0)$, which limits the recirculation zone close to the face of the bluff body. The streamlines clearly show the classical picture of an axisymmetric toroidal vortex. Figure 2 (right) shows a scheme of the global structure of the flow. The recirculation zone extends from the face of the bluff body to the stagnation point and around the toroidal vortex. It is also possible to distinguish a zero longitudinal velocity component surface, which delimits a region where negative values of $u$ are found only.

\section{Figure 2}

The flow similarity may be observed in Fig. 3(left), which shows for the three values of the Reynolds number, the velocity component, $u$, along the centerline $(r=0)$. It is evident that velocity exhibits negative values, first, decreasing to a point at $x \approx 0.45$ and then increasing toward the stagnation point located at $x \approx 0.92$. This justifies the subsequent analysis of the results of $\operatorname{Re}=45,400$ only. Additionally, it can be observed in Fig. 3(right) that the vortex center is always located at $(x, r)=(0.45,0.33)$. Note that, for spheres, the flow wake where self-similarity is observed is found for $x>50$ only [12]. In a far wake, the turbulent flow properties are functions of a single independent coordinate. However, even if the present results, obtained for the near wake, seem to exhibit a self-similar behavior, the measured large scale flow properties are found to be functions of two coordinates. 


\section{Figure 3}

In Fig. 4, where the average longitudinal and radial velocity components for $\operatorname{Re}=45,400$ are shown, a typical near wake behavior may be observed. Concerning the longitudinal velocity component, $u$, an abrupt velocity decrease may be observed at the bluff body rim, $r \approx 0.5$, thus generating a region of intense shear at the recirculation zone vicinity. Positive longitudinal velocity component values occur for $x>1$ due to the presence of the recirculation zone. The radial component $v$ is zero at the centerline and decreases with $r$. The highest $v$ absolute values are attained near the outer edge of the recirculation zone, representing a region of higher air entrainment toward the centerline. These maximum absolute values are similar to those characteristic of jet flows [12]. In this figure, this general trend is broken only at $x=0.17, r \leq 0.5$, where the flow is directed toward the rim of the bluff body. This occurs because this line crosses the bottom part of the vortex. For $r>0.5$, air entrainment toward the radial axis is always observed.

\section{Figure 4}

\subsection{Derived average properties}

The dimensional strain rate $S_{i j}$ and vorticity $W_{i j}$ are obtained from the average velocity fields according to $S_{i j}=0.5\left(\partial u_{j} / \partial x_{i}+\partial u_{i} / \partial x_{j}\right)$ and $W_{i j}=0.5\left(\partial u_{j} / \partial x_{i}-\partial u_{i} / \partial x_{j}\right)$. Not significant differences have been found with and without smoothing of the average velocity fields, so the latter is shown here. Following [21], the uncertainty associated to central differences of an average flow property is given by $0.707 \varepsilon_{U} / \Delta x_{i}$. The maximum strain rate uncertainty region is thus associated to the largest velocity uncertainty values. As a consequence, in this region the absolute uncertainty is $600 \mathrm{~s}^{-1}$. Figure 5 shows distributions of the strain rate components $S_{i j}$ and of the vorticity tensor components $w_{r x}$ calculated with a central difference scheme. The normal components $S_{x x}$ and $S_{r r}$ (left) exhibit similar distributions with opposed signs. The highest normal strain rate component absolute values are found at the vicinity of the stagnation points and at the bluff body rim. Two lobes with similar absolute values and an antisymmetric configuration are observed for $S_{r x}$ and $w_{r x}$, which is analogous to the behavior of near cylinder wakes [9]. These properties are nearly identically distributed because $\partial v / \partial x<\partial u / \partial r$. The corresponding maximum absolute values are thus found at the shear layer that originates at the bluff body rim. The local extreme closely follows the streamline that connects the bluff body rim to the $(x, r)=(0.92,0)$ stagnation point. This streamline is called the stagnation streamline. It is worth emphasizing that the measured fields of $s_{r x}$ and $w_{r x}$ are rather similar to those obtained by Braza et al. [9] for a cylinder near wake. Note that for the applications that are of interest to the authors 
[3], these high strain rates may simultaneously increase fuel air mixing and prevent non-premixed combustion stabilization. Indeed, if the averaged value of the strain rate is the inverse of a characteristic chemical time scale, combustion stabilization is unlikely.

\section{Figure 5}

\subsection{Reynolds stress tensor anisotropy}

The field distribution and the radial evolution of turbulent kinetic energy, $k$, are shown in Fig. 6. In this figure, it may be verified that $k$ exhibits a single lobe structure, with maximum values occurring around the $(x, r)=(0.92,0)$ stagnation point, i.e., the stagnation streamline. At the vicinity of the bluff body face, the values of $k$ are nearly independent of $r$ and are at least close to $r=0$ and $x<0.6$. Further downstream, the distribution of $k$ changes appreciably. Indeed, for $x \geq 1, k$ reaches a maximum at $r=0$ and decreases monotonically with $r$. Note that local maxima of $k$ are observed along the streamline that originates at the vicinity of the bluff body rim and reaches this stagnation point. This local distribution is similar to that noted above, corresponding to normal strain $S_{i i}$. It is interesting to verify that at the vicinity of the bluff body rim, the high $k$ values are associated with high shear rates, whereas $s_{r r}$ and $s_{x x}$ seem to be related to $k$ at the stagnation point.

\section{Figure 6}

The anisotropy tensor components $a_{i j}$ are given in Fig. 7. The anisotropy tensor is defined as $a_{i j}=\overline{u_{i}^{\prime} u_{j}^{\prime}}-2 / 3 \cdot \delta_{i j} \cdot k$, where $\overline{u_{i}^{\prime} u_{j}^{\prime}}$ are the Reynolds stress tensor components and $\delta_{i j}$ is the Kronecker delta $[11,28]$. This figure shows that with the exception of the trivial external air flow region $(x>0.6)$, significant departures from isotropy occur. Indeed, the normal components are not uniform throughout the wake region, for instance, $a_{x x}$ exhibits values exceeding 0.009 at the shear layer, which originates at the bluff body rim and are smaller than -0.012 at the $(0.92,0)$ stagnation point. A nearly opposite behavior is found for $a_{r r}$. Note that the $a_{i i}$ maximum absolute values arise around the $(0.92,0)$ stagnation point similar to the normal strain rate components. The $a_{r x}$ component is zero at the symmetry line, as expected. It is also worth noting that the region of $a_{r x}$ extrema -0.015 seems to correspond with the stagnation streamline flow behavior that goes from being shear dominated to normal stress dominated. This extreme value is similar to that found at the fully developed self-similar sphere wake region [12]. It should be noted that this near wake behavior is somewhat similar to that found at near cylinder wakes by Braza et al. [9], even if alternate and periodical vortex shedding is absent here. Braza et al. [9] have determined that the periodic motion 
associated with cylinder vortex shedding leads to extreme Reynolds stress values that are an order of magnitude larger than those measured in the present wake.

\section{Figure 7}

\subsection{Anisotropy and Lumley triangle}

Further characterization of the turbulent state is achieved through the theory of Lumley [29], which involves the normalized anisotropy tensor $b_{i j}$ and the associated tensor invariants $I_{n}$. The normalized anisotropy tensor, defined as $b_{i j}=a_{i j} / 2 k$, is a zero trace tensor and, hence, has only two independent invariants, i.e., the traces of $b_{i j}^{2}$ and $b_{i j}^{3}$ matrices, i.e., $I I=b_{i j} b_{j i}$ and $I I I=b_{i j} b_{j k} b_{k i}$, respectively [11,28]. Using the classical graphical representation of the turbulent states in $(I I I,-I I)$ coordinates, it may be shown that the turbulent state realizable region is a triangle within which are situated all possible $b_{i j}$ values. The triangle boundaries match particular turbulent states. The turbulent state may thus be characterized by means of the invariants only. Furthermore, to estimate the local degree of departure from isotropy, a single parameter, $R=\sqrt{(I I I)^{2}+(-I I)^{2}}$, is used to measure the distance from the isotropic state $R=0$ [19]. One of the key simplifications in Lumley's anisotropy analysis is that it does not make a distinction between large and small scales. It is well known that the largest scales are inherently 3D and anisotropic, but it is unclear how this proceeds into the small scales towards the Kolmogorov region. Unfortunately, given the estimated Kolmogorov frequency $7.5 \mathrm{kHz}$, and the maximum frequency which the $\mathrm{TiO}_{2}$ particles are expected to follow, $5.4 \mathrm{kHz}$, it seems impossible to accurately use the present data in order to decompose the movement into large and small scales. A similar invariant treatment is performed with the strain rate tensor, which is also a zero trace tensor. It is worth recalling that classical eddy viscosity models are often based on a direct proportionality between $b_{i j}$ and $S_{i j}$

The invariant fields of $b_{i j}$ and $s_{i j}$ are shown in Fig. 8. The corresponding distance to isotropy ( $R$ and $R_{s}$ ) is given in Fig. 9. Considering the fields of $-I I$ and $I I I$ first, it may be observed that some of the local maximum values closely follow the stagnation streamline. As this streamline approaches the $(0.92,0)$ stagnation point, $-I I$ values decrease, albeit remaining positive, whereas III becomes negative. Within the recirculation region, local extrema also arise. Indeed, - II and III reach 0.06 and 0.04 close to the toroidal vortex center. Concerning the strain rate invariants, $-I I_{s}$ and $I I I_{s}$, again local extrema arise around the stagnation streamline, which 
underscores the turbulence generation mechanism. However, the $-I I_{s}$ and $I I I_{s}$ behavior deviates significantly from that of $-I I$ and $I I I$, both at the vicinity of the $(0.92,0)$ stagnation point and within the vortex. Indeed, both $-I I_{s}$ and $I I I_{s}$ decrease to similar positive values at these regions, and a direct relation to $-I I$ and $I I I$ is not observed. The corresponding distance to isotropy, $R$ and $R_{s}$, is accordingly affected.

\section{Figure 8}

\section{Figure 9}

To follow the evolution of the tensor invariants, Fig. 10 shows the associated behavior along three different streamlines. The first of these streamlines originates at $(x, r)=(0.23,1)$ and is represented in Fig. 10 by the a0-a1 path. Because this streamline traverses a nearly isotropic region, small values of $-I I$ and $I I I$ are observed $(R<0.01)$ and $-I I_{s} \approx I I I_{s} \approx 0$. The origin of the second streamline $(x, r)=(0.23,0.6)$ is chosen to represent a fluid particle trajectory that remains outside the recirculation region, but under its influence, is noted as b0-b1. Along this path, $-I I_{s}$, $I I I_{s}$ and $R_{s}$ first increase, reaching maximum values of $0.8,0.26$ and 0.84 , respectively, and then decrease along the most right boundary of the triangle. A similar behavior is observed for the anisotropy tensor invariants, $-I I$ and $I I I$. However, this path is internal to the triangle. The third path, $\mathrm{c} 0-\mathrm{p}-\mathrm{c} 1$, represents the stagnation point streamline behavior. As noted above, - II and III , first increase (up to $x \sim 0.75$ ) then decrease. These increasing/decreasing paths are internal to the triangle and seem to follow a straight line (at least within the limits of the experimental uncertainty). It is worth emphasizing that upon reaching the stagnation point (noted p), the turbulent flow progressively returns to isotropy further downstream $(x>1)$, strictly following the axisymmetric compression boundary. Along the $\mathrm{c} 0-\mathrm{p}-\mathrm{c} 1$ path, the turbulence state is gradually modified from an initial state ( $\mathrm{c} 0$ ) within the triangle to a disk-like turbulence (p), tending to an isotropic state (c1). The corresponding path at the $\left(-I I_{s}, I I I_{s}\right)$ plane is rather different. Indeed, both these invariants simultaneously increase then for $x>0.84$ and decrease along the right triangle boundary. Finally, the $q$ in Fig. 10 indicates the invariant values observed at the vortex center.

\section{Figure 10}




\section{Concluding Remarks}

This work involved a characterization of the isothermal turbulent flow field at the self-confined near wake of a circular bluff body. Such a near wake appears to have received little attention in the past, at least from the standpoint if turbulence anisotropy behavior. The main conclusions of this study are as follows:

- The average velocity and components are consistent with those of planar and cylinder wakes [12], and exhibit similarity with respect to the Reynolds number.

- Turbulent kinetic energy is nearly constant at the immediate vicinity of the bluff body surface and exhibits local maximum at the shear layer originating at the bluff body rim. The highest values are found further downstream at the vicinity of the stagnation point, which is similar to what occurs at near cylinder wakes [9].

- Reynolds stress tensor component anisotropy is significant both near the stagnation point and at the shear layer. The former seems to be controlled by the normal strain rate, whereas the latter is related to high shear rates.

The measured extreme values of the Reynolds stress components are an order of magnitude smaller than those characteristic of cylinder wakes [9]. This is attributed to the absence of vortex shedding here.

- The Reynolds stress tensor invariants are related to the strain rate tensor invariants at the flow regions where shear dominates. Close to the stagnation point and at the vortex center vicinity, this relation is not observed.

- Following a streamline that reaches the freely standing stagnation point, anisotropy first increase with $I I I>0$, then decrease to negative values of $I I I$, at this stagnation point. Departing from this freely standing stagnation point, and following this streamline further downstream, the anisotropy decreases along the axisymmetric contraction boundary of the Lumley triangle.

Future work will examine how the Reynolds stress tensor anisotropy components and the corresponding invariants are affected by the presence of turbulent flames in different combustion modes. Indeed, a previous investigation [3] has shown that intermittent combustion heat release at the wake influences the distribution of turbulent kinetic energy. In particular, fluctuating turbulent non-premixed flames exhibited an increase of turbulent kinetic energy. Such a situations is outside the domain of validity of classical combustion models. It is thus expected that understanding the influence of the combustion process on the turbulence anisotropy would lead to improved combustion models.

\section{Acknowledgments}

This research has been supported by Capes/Pro-Engenharias and Petrobras under the technical monitoring of Dr. Ricardo Serfaty (Project: Development of a modeling technique for turbulent combustion based on an Eulerian/Lagrangian approach - Phase II, Contract No.: 
0050.0080122.12.9). During this work Luís Fernando Figueira da Silva was on leave from the Institut Pprime (Centre National de la Recherche Scientifique, France).

\section{References}

1. Masri, A.R., Dibble, R.W., Barlow, R.S.: The structure of turbulent nonpremixed flames revealed by Raman-Rayleigh-LIF measurements. Prog. Energy Combust Sci. 22(4), 307-362 (1996). doi:10.1016/S0360-1285(96)00009-3

2. Egúsquiza, J.C., Figueira da Silva, L.F.: Turbulent non-premixed ethanol-air flame experimental study using laser diagnostics. J. Braz. Soc. Mech. Sci. Eng. 35(3), 177-188 (2013). doi:10.1007/s40430-013-0017-y

3. Caetano, N.R., Figueira da Silva, L.F.: A comparative experimental study of turbulent non premixed flames stabilized by a bluff-body burner. Exp. Therm. Fluid Sci. 63, 20-33 (2015). doi:10.1016/j.expthermflusci.2015.01.006

4. Andrade, F.O., Figueira da Silva, L.F., Mura, A.: Large eddy simulation of turbulent premixed combustion at moderate Damköhler numbers stabilized in a high-speed flow. Combust. Sci. Technol. 183(7), 645-664 (2011). doi:10.1080/00102202.2010.536600

5. Celis, C., Figueira da Silva, L.F.: On mass consistency techniques used in LES/PDF simulations of turbulent reacting flows. In: Eighth Mediterr. Combust. Symp, Izmir (2013)

6. Vedovoto, J.M., Silveira Neto, A., Figueira da Silva, L.F., Mura, A.: Influence of synthetic inlet turbulence on the prediction of low Mach number flows. Comput. Fluid 106, 135-153 (2015). doi:10.1016/j.compfluid.2014.09.046

7. Durao, D. F.G., Whitelaw, J.H.: Velocity characteristics of the flow in the near wake of a disk. J. Fluid Mech. 85(2), 369-385 (1978). doi:10.1017/s0022112078000683

8. Huang, R.F., Lin, C.L.: Velocity field of a bluff-body wake. J. Wind Eng. Ind. Aerodyn. 85(1), 31-45 (2000). doi:10.1016/S0167-6105(99)00117-8

9. Braza, M., Perrin, R., Hoarau, Y.: Turbulence properties in the cylinder wake at high Reynolds number. J. Fluids Struct. 22(6-7), 757-771 (2006). doi:10.1016/j.jfluidstructs.2006.04.021

10. Bitter, M., Scharnowski, S., Hain, R., Kahler, C.J.: High-repetition-rate PIV investigations on a generic rocket model in sub- and supersonic flows. Exp. Fluids 50(4), 1019-1030 (2011). doi:10.1007/s00348-010-0988-8

11. Pope, S.B.: Turbulent Flows. Cambridge University Press, Cambridge (2000)

12. Chassaing, P.: Turbulence en mécanique des fluides: analyse du phénomène en vue de sa modélisation à l'usage de l'ingénieu. Cépaduès - éditions, Paris (2000)

13. Uberoi, M.S., Freymuth, P.: Turbulent energy balance and spectra of the axisymmetric wake. Phys. Fluids 13, 2205-2210 (1970). doi:10.1063/1.1693225

14. Redfor, J.A., Castro, I.P., Coleman, G.N.: On the universality of turbulent axisymmetric wakes. J. Fluid Mech. 710, 419-452 (2012). doi:10.1017/jfm.2012.371

15. Herrin, J.L., Dutton, J.C.: Supersonic base flow experiments in the near wake of a cylindrical afterbody. AIAA J. 32(1), 77-83 (1994). doi:10.2514/3.11953 
16. Antonia, R.A., Zhou, T., Romano, G.P.: Small-scale turbulence characteristics of twodimensional bluff body wakes. J. Fluid Mech. 459, 67-92 (2002). doi:10.1017/S0022112002007942

17. Thiesset, F., Danaila, L., Antonia, R.A.: Dynamical effect of the total strain induced by the coherent motion on local isotropy in a wake. J. Fluid Mech. 720, 393-423 (2013). doi:10.1017/jfm.2013.11

18. Choi, K., Lumley, J.L.: The return to isotropy of homogeneous turbulence. J. Fluid Mech. 436, 59-84 (2001). doi:10.1017/S002211200100386X

19. Derksen, J.J., Doelman, M.S., Van den Akker, H.E.: Three-dimensional LDA measurements in the impeller region of a turbulently stirred tank. Exp. Fluids 27(6), 522-532 (1999). doi:10.1007/s003480050376

20. Hartmann, H., Derksen, J.J., Montavon, C., Pearson, J., Hamill, I.S., van den Akker, H.E.A.: Assessment of large eddy and RANS stirred tank simulations by means of LDA. Chem. Eng. Sci. 59(12), 2419-2432 (2004). doi:10.1016/j.ces.2004.01.065

21. Raffel, M., Willert, C., Wereley, S., Kompenhans, J.: Particle Image Velocimetry: A Practical Guide. Springer, Berlin (2007)

22. Roesgen, T.: Optimal subpixel interpolation in particle image velocimetry. Exp. Fluids 35(3), 252-256 (2003). doi:10.1007/s00348-003-0627-8

23. Adrian, R., Westerweel, J.: Particle Image Velocimetry. Cambridge University Press, New York (2011)

24. Sciacchitano, A., Neal, D.R., Smith, B.L., Warner, S.O., Vlachos, P.P., Wieneke, B., Scarano, F.: Collaborative framework for PIV uncertainty quantification: comparative assessment of methods. Meas. Sci. Technol. 26(7), 074004 (2014). doi:10.1088/0957-0233/26/7/074004

25. Benedict, L.H., Gould, R.D.: Towards better uncertainty estimates for turbulence statistics. Exp. Fluids 22(2), 129-136 (1996). doi:10.1007/s003480050030

26. Lazar, E., DeBlauw, B., Glumac, N., Dutton, C., Elliott, G.: A practical approach to PIV uncertainty analysis. In: 27th AIAA Aerodynamic Meas. Technol. Ground Testing Conf., Chicago (2010)

27. Melling, A.: Tracer particles and seeding for particle image velocimetry. Meas. Sci. Technol. 8(12), 1406-1416 (1997). doi:10.1088/0957-0233/8/12/005

28. Durbin, P.A., Pettersson Reif, B.A.: Statistical Theory and Modeling for Turbulent Flows. John Wiley \& Sons, Chichester (2011)

29. Lumley, J.L.: Computational modelling of turbulent flows. Adv. Appl. Mech. 18, 123-176 (1978). doi:10.1016/S0065-2156(08)70266-7 


\section{Figure Captions}

Fig. 1 Experimental setup, bluff body scheme and dimensions

Fig. 2 Flow structure average longitudinal velocity component field, $u(\mathrm{~m} / \mathrm{s})$ (left), streamlines and velocity vectors, $\operatorname{Re}=45,400$, and scheme of the mean flow structure (right)

Fig. 3 Longitudinal evolution of the average velocity component, $u$, at centerline $r=0$ (left) and at row $r=0,33$ (right); i.e., passing through the vortex center

Fig. 4 Radial evolution of the average velocity components, $u$ (right) and $v$ (left)

$\mathrm{V}_{\mathrm{x}} / \mathrm{V}_{\mathrm{y} \_ \text {max }} \mathrm{V}_{\mathrm{y}} / \mathrm{V}_{\mathrm{y} \_ \text {max }}$ for different longitudinal positions, $\mathrm{Re}=45,400$

Fig. 5 Components strain rate tensor $s_{r r}, s_{x x}$, (left) and $s_{r x}$ and vorticity $s_{r x}$ (right).

Streamlines in white, $\operatorname{Re}=45,400$

Fig. 6 Turbulent kinetic energy, $k$, fields and profiles. Streamlines in white, $\operatorname{Re}=45,400$

Fig. 7 Anisotropy tensor components $a_{i j}$ fields. Streamlines in white, $\operatorname{Re}=45,400$

Fig. 8 Field of the $-I I$ and $I I I$ invariants for $b_{i j}$ (left) and $S_{i j}$ (right), streamlines in white

Fig. 9 Distance from the isotropic state, $R$ (left) and $R_{s}$ (right), streamlines in white

Fig. 10 Lumley invariants' distribution of the anisotropy tensor $b_{i j}\left(\right.$ left), and strain rate tensor $S_{i j}$ (right). Superimposed are the paths corresponding to streamlines starting at $r=1$ (a0-a1), $r=0.6$ (a0-a1) and the stagnation streamline $r=0.5$ (c0-p-c1) 

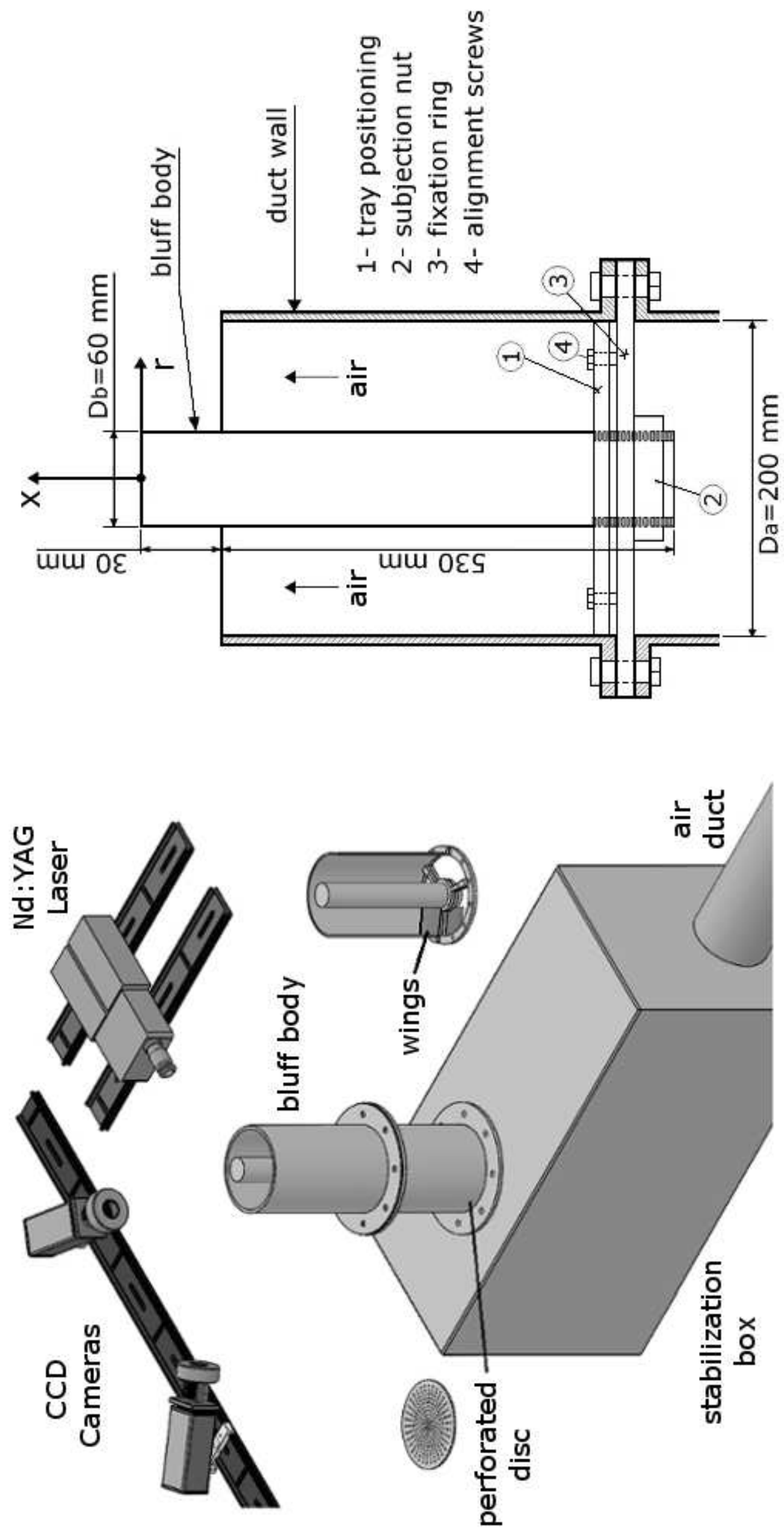

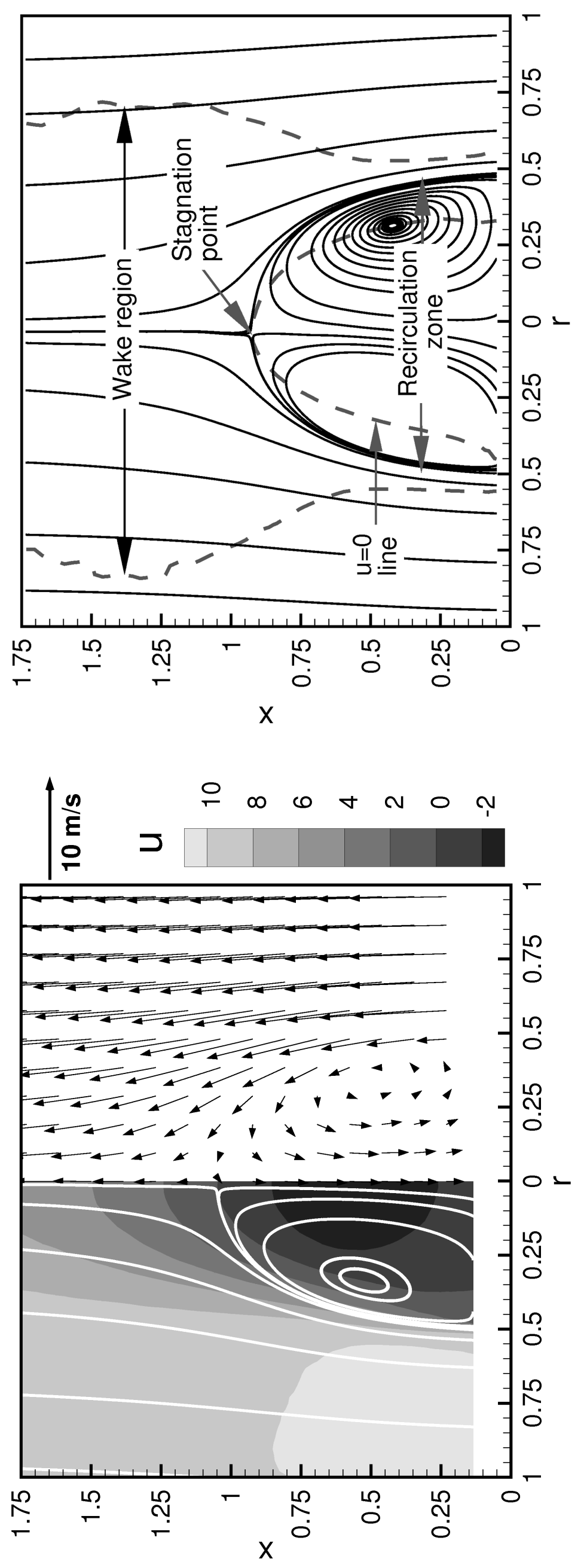

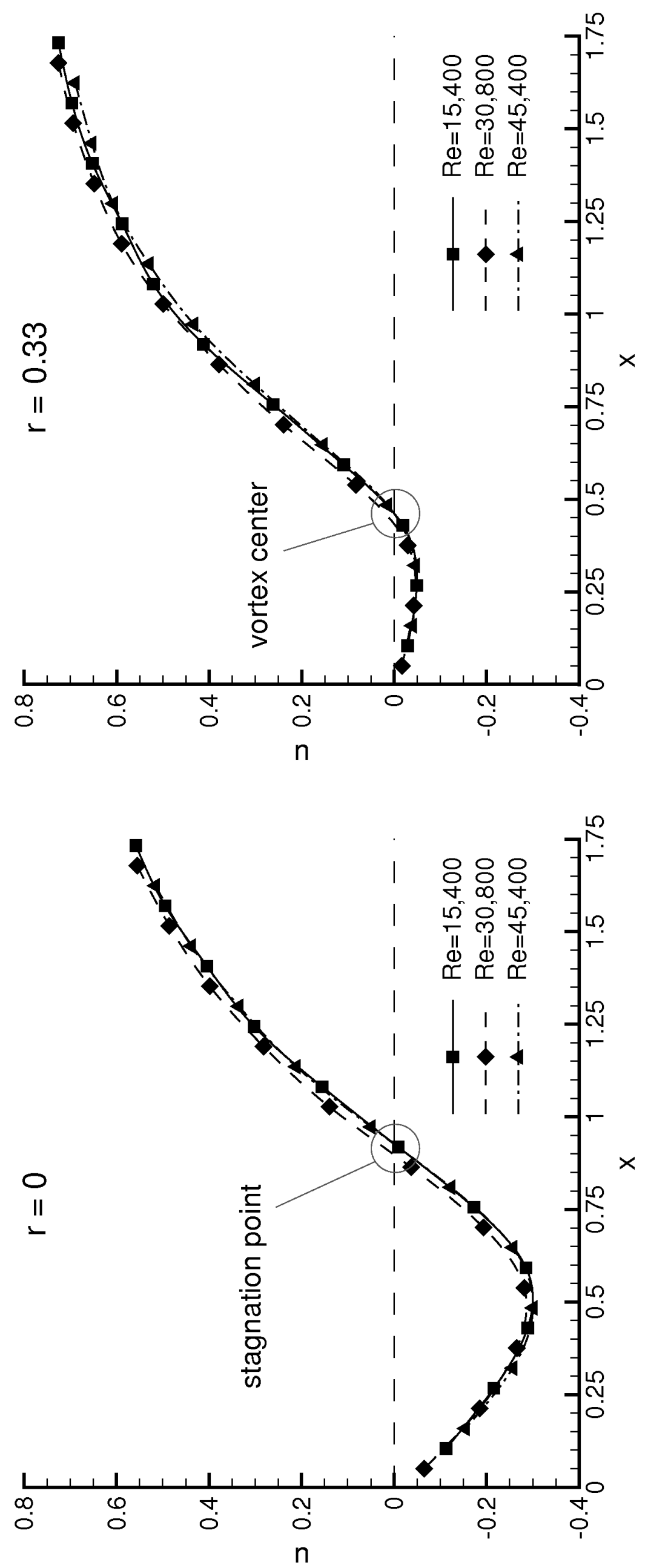

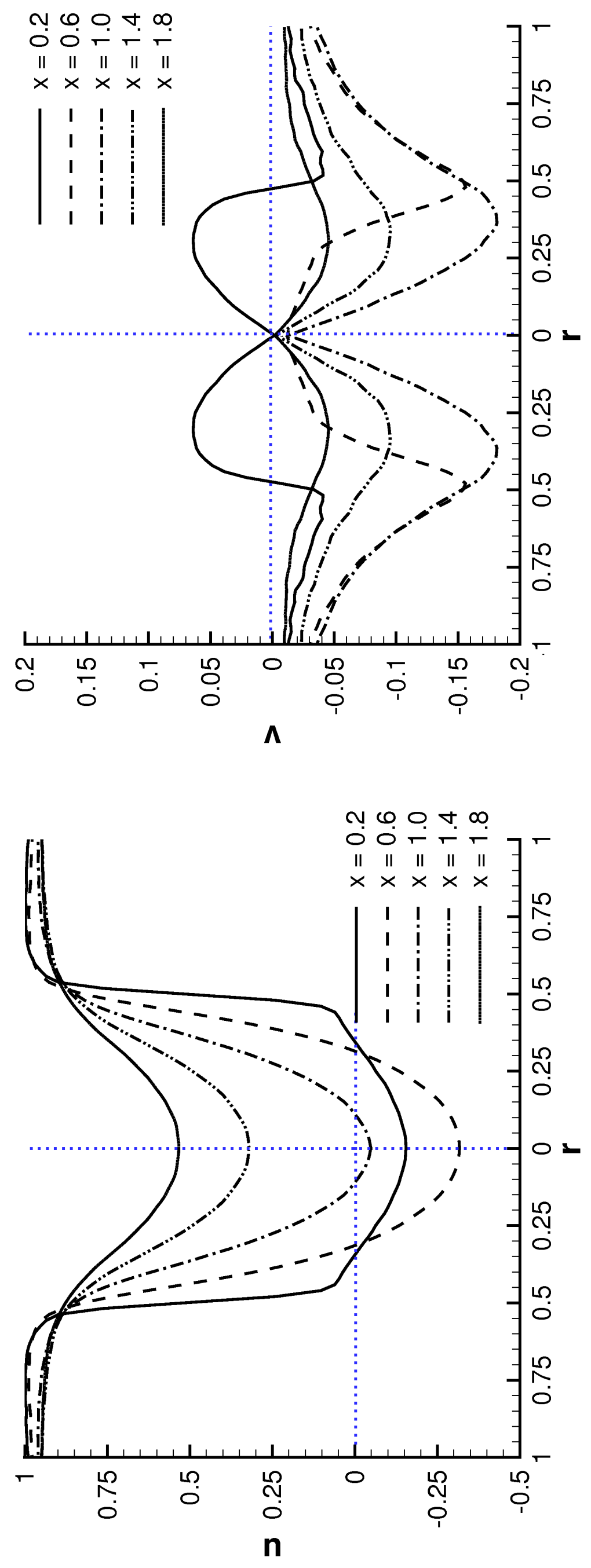

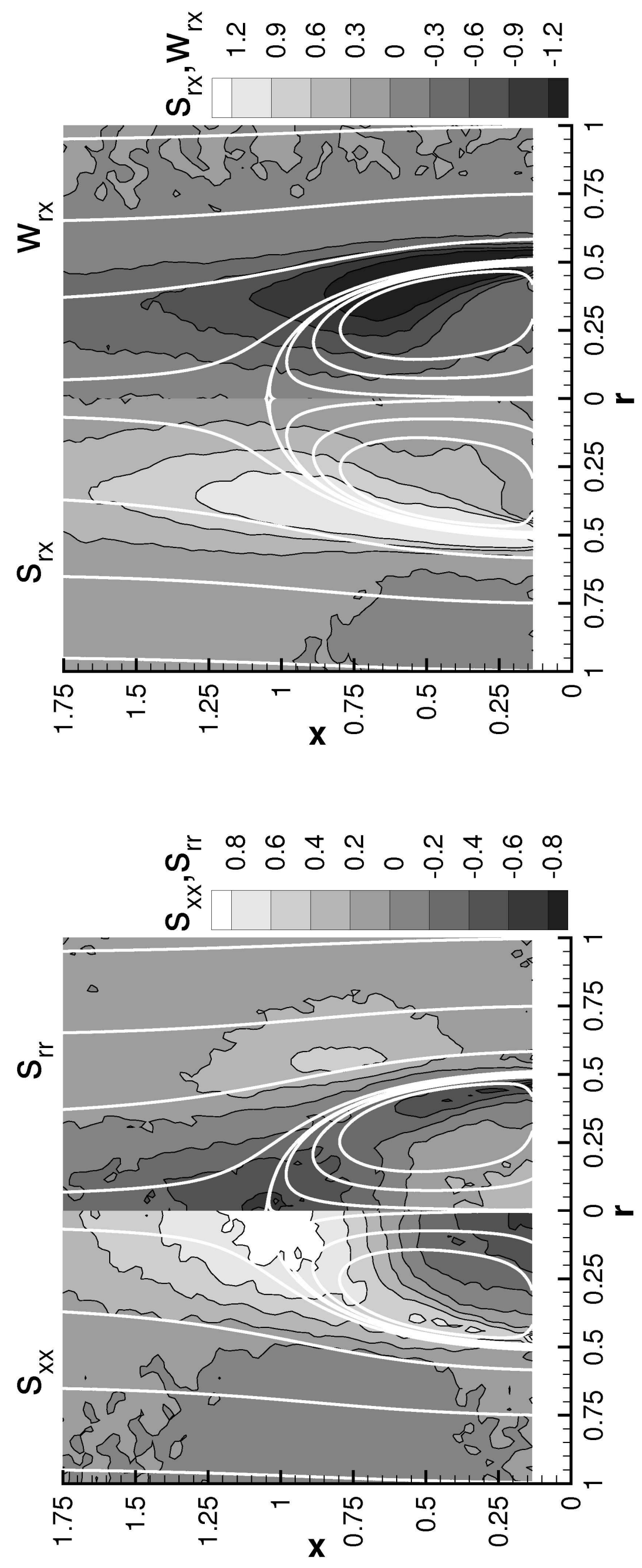


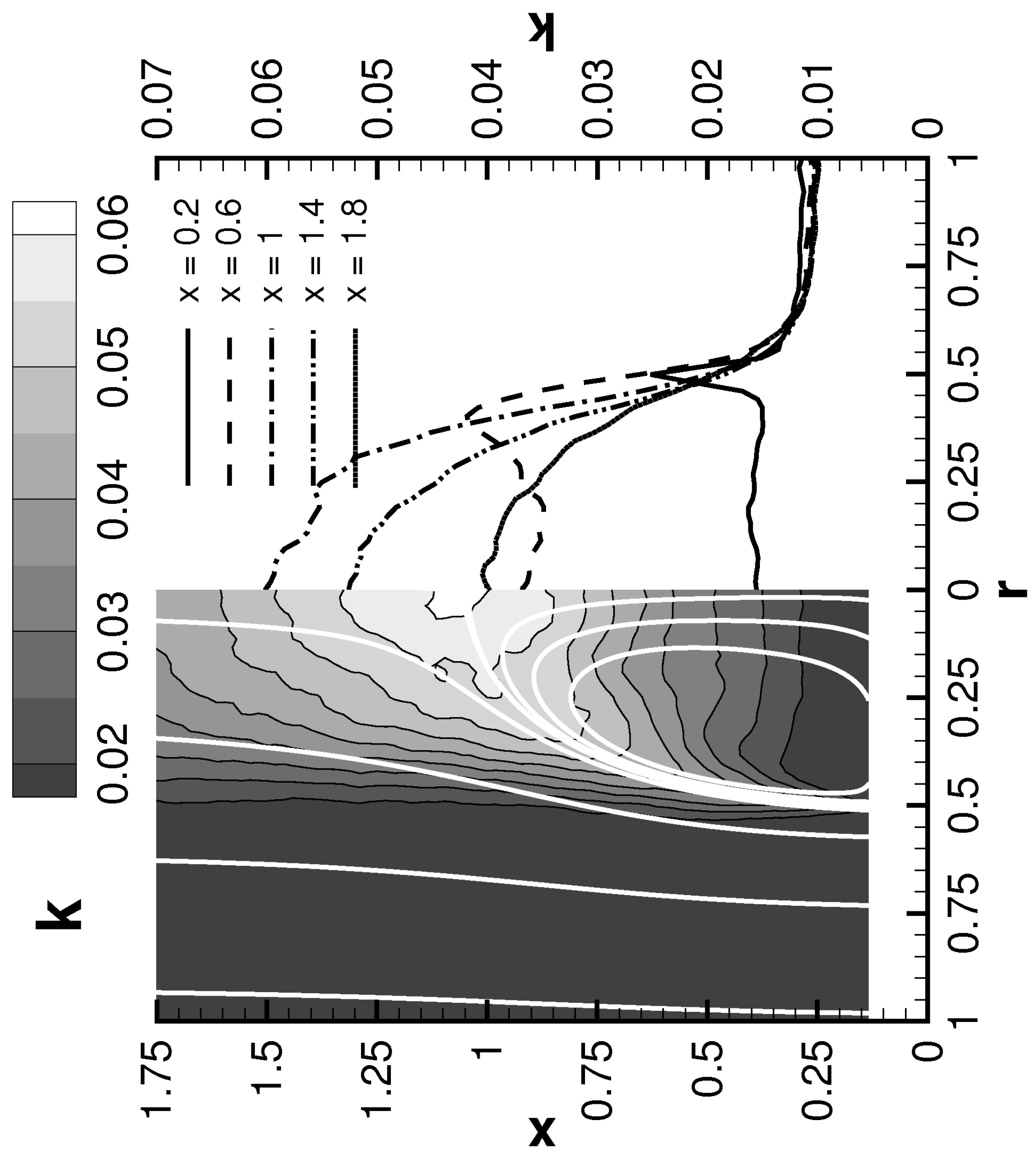




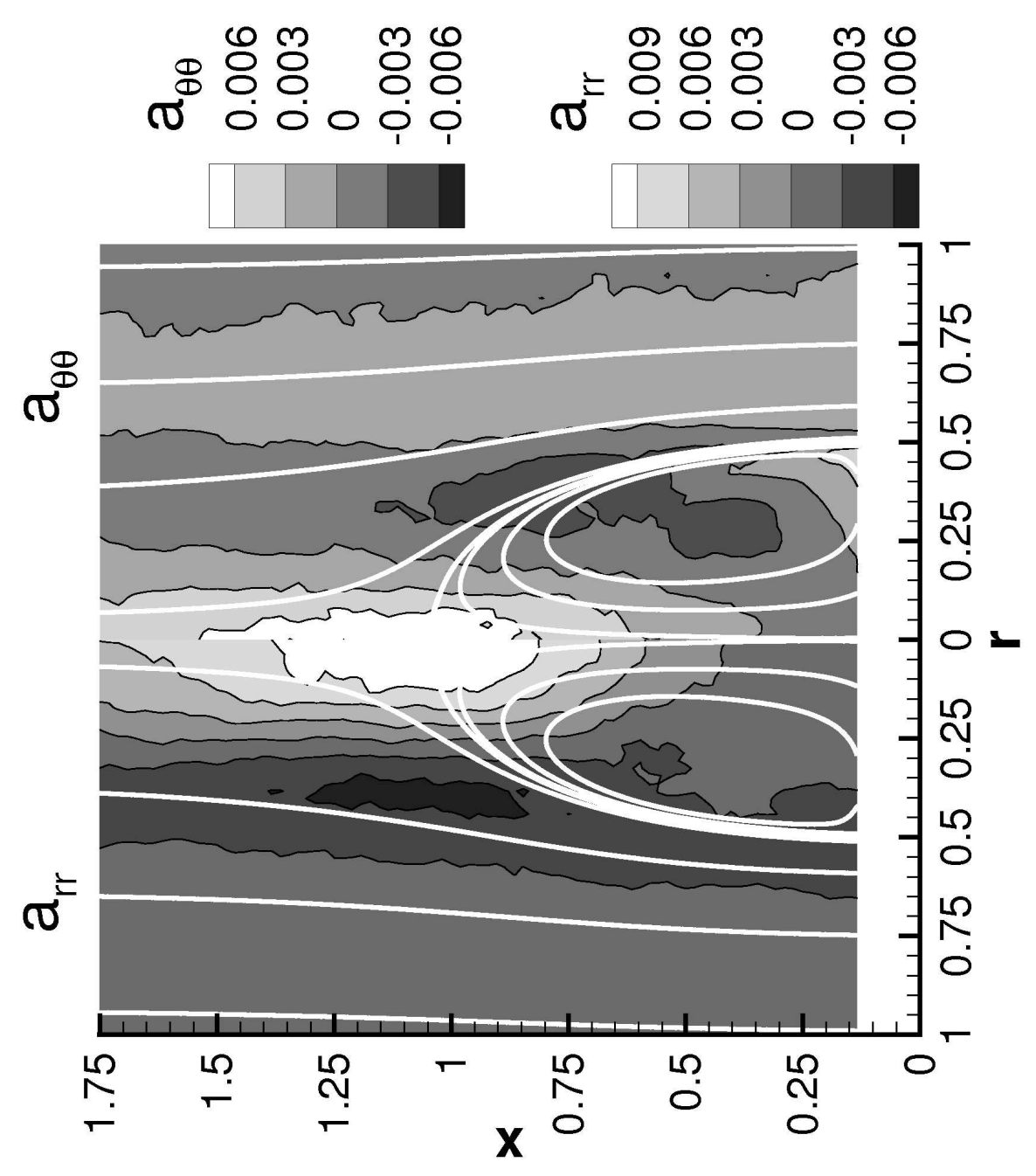

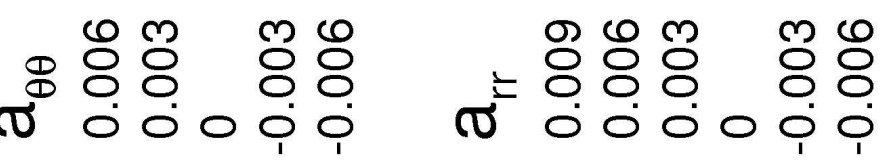

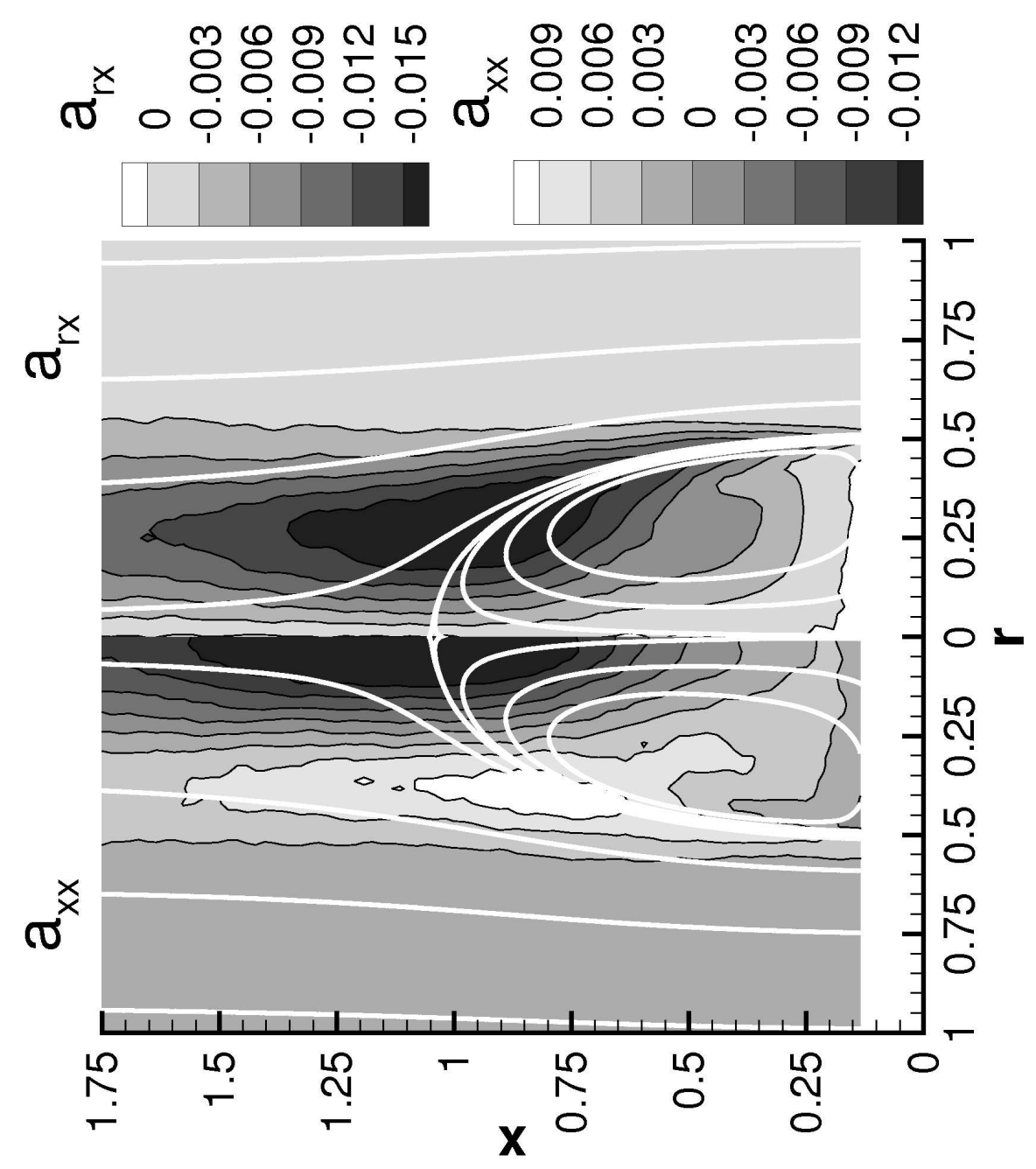



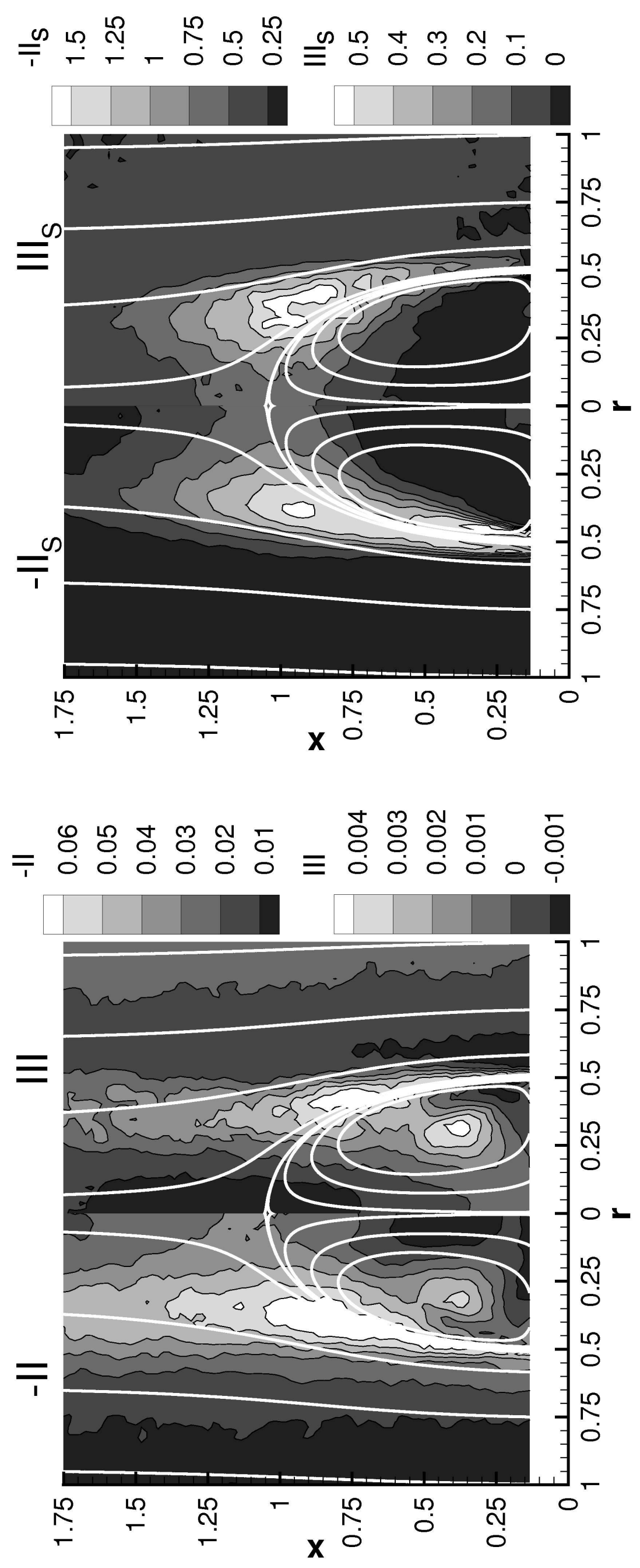


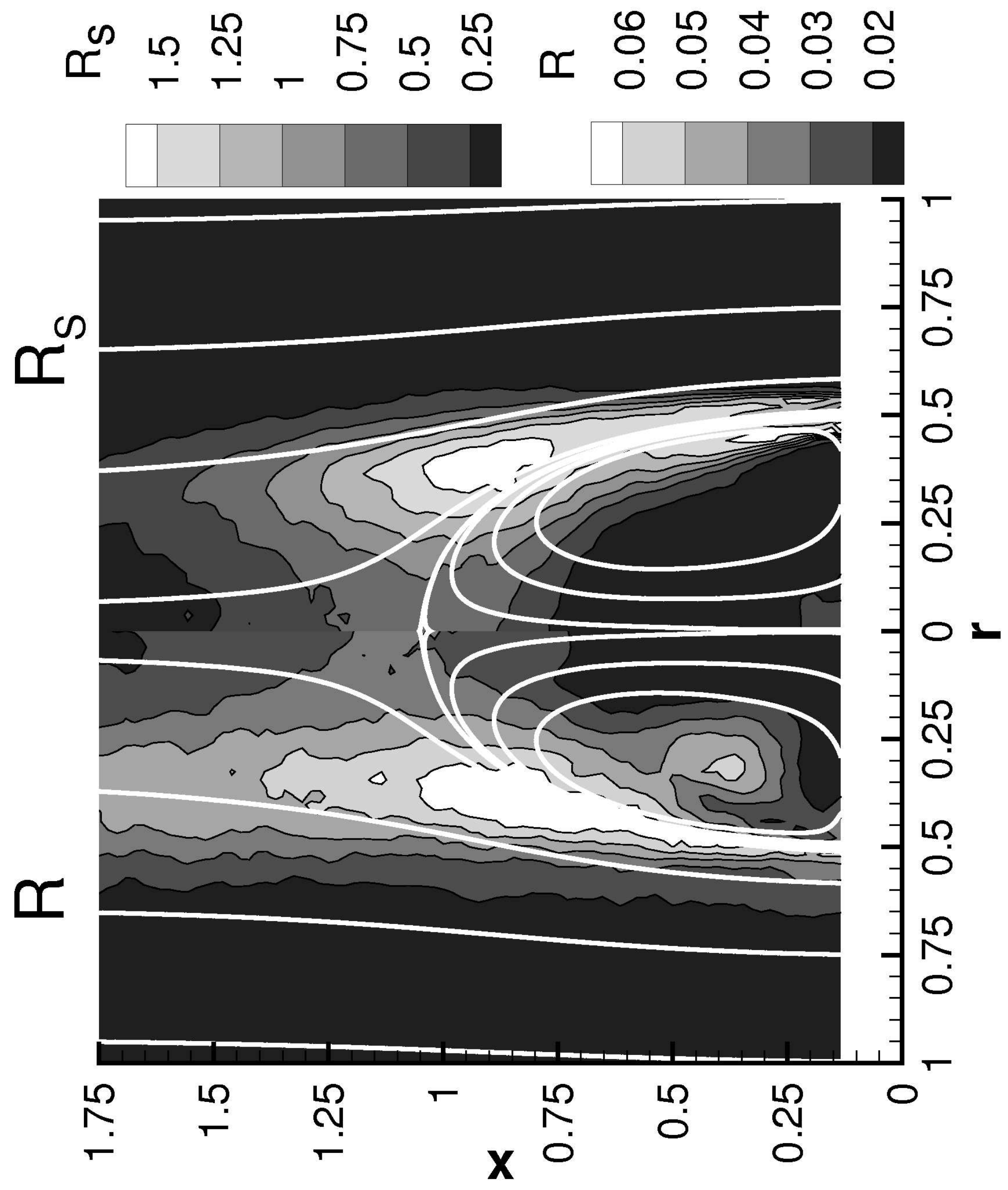



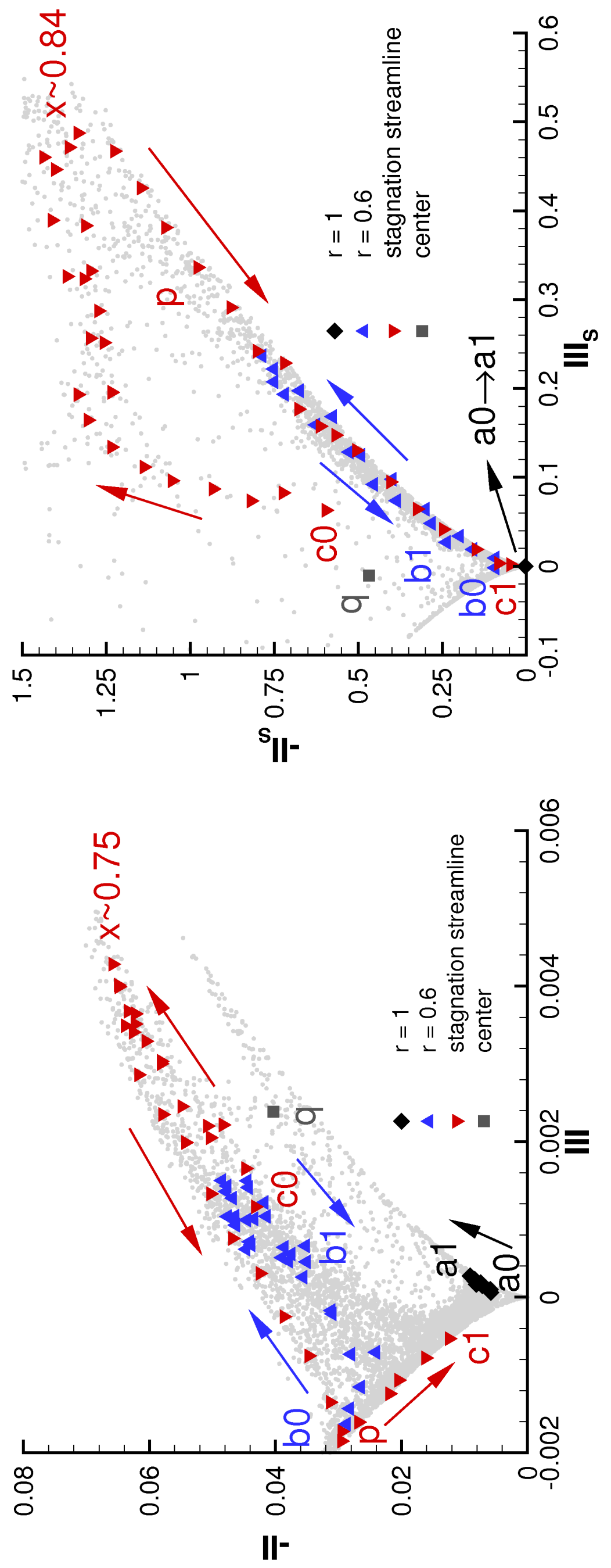